\title{
GEOLOGICAL SURVEY OF FINLAND RADIOCARBON MEASUREMENTS VII
}

\author{
AULIS HEIKKINEN and OSMO ÄIKÄ̈̈ \\ ${ }^{14} \mathrm{C}$ Laboratory, Geological Survey of Finland \\ SF-02150 Espoo 15, Finland
}

This list of measurements includes most of the geologic samples dated in this laboratory since the publication of our last list $(\mathrm{R}, 1974$, v 16, p 252-268).

The age calculations are based on $95 \%$ of the isotopically corrected activity of the NBS oxalic acid standard and on a halflife of $5568 \mathrm{yr}$. The results are reported in years before 1950 . Most of the samples have been corrected for deviations from the normal ${ }^{13} \mathrm{C} /{ }^{12} \mathrm{C}$ ratios $\left(\delta^{13} \mathrm{C}=\right.$ $-25 \%$ in the PDB scale). The $\delta^{13} \mathrm{C}$ values quoted are relative to the PDB standard.

We measured the ${ }^{14} \mathrm{C}$ activity of the $\mathrm{CO}_{2}$ gas in proportional counters, as described by Heikkinen et al, 1974. Each sample was counted at least twice for a period of not less than $2400 \mathrm{~min}$. When measured activity was low, alternating counting sample/background was applied to the sample.

\section{ACKNOWLEDGMENTS}

The descriptions of the samples are based on data provided by collectors and submitters. Sincere thanks are due the contributors who helped in the preparation of the text. Our special gratitude goes to $\mathrm{R}$ Ryhage, Karolinska Inst, Stockholm, and his staff for the ${ }^{13} \mathrm{C} /{ }^{12} \mathrm{C}$ measurements. The authors are indebted to Anna-Kristiina Koivisto, Sirkka Hongisto, and Pirjo Hakala for laboratory work.

\section{SAMPLE DESCRIPTIONS}

\section{GEOLOGIC SAMPLES \\ Finland}

\section{Marrasjärvi series, Rovaniemi, N Finland}

Four samples from lowermost part of till bed underlain by sorted sediments. Humus content $0.8 \%$, Marrasjärvi, Raumo sand pit $\left(66^{\circ} 53^{\prime}\right.$ $\mathrm{N}, 25^{\circ} 10^{\prime} \mathrm{E}$ ), surface alt $125 \mathrm{~m}$, depth $2.5 \mathrm{~m}$. Coll and subm May 1973 by Aulis Heikkinen and Raimo Kujansuu. The fibrous matter, which may have been of postglacial age, was picked out of samples in the lab.

Su-236. Marrasjärvi

$$
\delta^{13} \mathrm{C}=-28.5 \%
$$

Sample, 1430g, boiled $1 \mathrm{~N} \mathrm{HCl}$. Organic material in till analyzed (10 2-day counts).

\section{Su-237. Marrasjärvi}

Sample weight $1300 \mathrm{~g}$, subjected to non-chemical processing (10 2-day counts). 


\section{Su-263. Marrasjärvi}

$$
\begin{array}{r}
>\mathbf{5 4 , 0 0 0} \\
\delta^{13} \mathrm{C}=-26.5 \% \text { 。 }
\end{array}
$$

Sample weight $3290 \mathrm{~g}$. Humic acids analyzed. Preparation: $1 \mathrm{~N}$ $\mathrm{NaOH}$ dissolution, filtrate $+\mathrm{HCl}=$ humic acids (4 2-day counts).

\section{Su-264. Marrasjärvi}

$26,300 \pm 250$

$$
\delta^{13} C=-26.5 \%
$$

Same as Su-263. Residue boiled in $1 \mathrm{~N} \mathrm{HCl}$, residue of organic material in till analyzed (4 2-day counts).

\section{Su-265. Marrasjärvi}

$$
\begin{array}{r}
>\mathbf{4 9 , 0 0 0} \\
\delta^{13} \mathrm{C}=-28.0 \%
\end{array}
$$

Sample weight $2990 \mathrm{~g}$. Humic acids analyzed. Preparation: $0.5 \mathrm{~N}$ $\mathrm{NaOH}$ dissolution, filtrate $+\mathrm{HCl}=$ humic acids (3 2-day counts).

\section{Su-266. Marrasjärvi}

$$
\begin{aligned}
&>\mathbf{4 0 , 7 0 0} \\
& \delta^{1 s} C=-27.7 \% \circ
\end{aligned}
$$

Same as Su-265. Residue of organic material in till analyzed (3 2-day counts. Comments: 1) because ${ }^{14} \mathrm{C}$ activity in samples may be caused by contamination, estimated age of organic substances transported by glacial ice is $>55,000 \mathrm{yr}$ (Heikkinen, 1975). 2) according to stratigraphy, glacial flow directions and pollen content of basal till layer, the esker at Marrasjärvi was deposited during Early-Weichselian deglaciation and covered with till during Late-Weichselian glaciation (Kujansuu, 1975).

\section{Su-289. Savukoski, N Finland}

$9300 \pm 160$

Peat and clayey silt from lower part of postglacial peat deposit, depth 1.3 to $1.45 \mathrm{~m}$, surface alt $219 \mathrm{~m}$, Sokli $\left(67^{\circ} 49^{\prime} \mathrm{N}, 29^{\circ} 24^{\prime} \mathrm{E}\right)$. Coll 1972 and subm 1973 by E Ilvonen.

\section{Su-503. Savukoski, N Finland}

Diatomaceous earth deposited in fresh water, depth 1.1 to $1.2 \mathrm{~m}$ surface alt $252 \mathrm{~m}$, Sokli $\left(67^{\circ} 48^{\prime} \mathrm{N}, 29^{\circ} 20^{\prime} \mathrm{E}\right)$. Coll 1973 and subm 1975 by $\mathrm{E}$ Ilvonen. Comment: sample derives from a diatomaceous earth deposit between 2 till beds. Microfossil composition suggests that sample is interglacial in age.

\section{Su-290. Artjärvi, S Finland}

$$
960 \pm 50
$$

Gyttja clay taken with piston corer. Sample depth 0.6 to $0.7 \mathrm{~m}$, water depth $65 \mathrm{~m}$, from lake Pyhäjärvi $\left(60^{\circ} 40^{\prime} \mathrm{N}, 26^{\circ} 02^{\prime} \mathrm{E}\right)$. Coll and subm 1973 by E Kukkonen and R Tynni. Comment: increase in Secale pollen in Artjärvi area. Oldest Secale pollen, according to annual microvarves, AD 750, at level $-1.3 \mathrm{~m}$ (Kukkonen \& Tynni, 1970).

\section{Pieni Kankaanlampi series, Kemijärvi, N Finland}

Samples from various levels of waterside bog at Lake Pieni Kankaanlampi $\left(66^{\circ} 43^{\prime} \mathrm{N}, 27^{\circ} 56^{\prime} \mathrm{E}\right)$, surface alt $160.5 \mathrm{~m}$. Coll 1972 with piston sampler and subm 1973 by E Lappalainen. 
Ooze taken from 5.60 to $5.68 \mathrm{~m}$ below bog surface. Comment: pollen analysis shows transition from Betula to Pinus maximum.

Su-293. Pieni Kankaanlampi

$10,470 \pm 100$ $\delta^{13} \mathrm{C}=-36.5 \%$

Ooze, depth 6.22 to $6.29 \mathrm{~m}$. Comment: pollen analyses, Betula maximum.

Su-294. Pieni Kankaanlampi

$11,400 \pm 200$

Ooze, depth 6.32 to $6.40 \mathrm{~m}$. Comment: Betula maximum.

\section{Su-300. Laukaa, Central Finland}

$6630 \pm 110$
$\delta^{13} C=-27.5 \%$

From peat above sand, taken with piston sampler at depth 1.66 to $1.70 \mathrm{~m}$, surface alt $134 \mathrm{~m}$, Suojärvenneva bog $\left(62^{\circ} 37^{\prime} \mathrm{N}, 25^{\circ} 58^{\prime} \mathrm{E}\right)$. Coll 1973 by A Leino and subm 1973 by V E Valovirta.

\section{Su-301. Laukaa, Central Finland}

$$
\begin{array}{r}
\mathbf{8 9 0 0} \pm \mathbf{1 0 0} \\
\delta^{13} \mathrm{C}=-27.7 \% \text { o }
\end{array}
$$

From peat above sand, taken with piston sampler, depth 2.76 to $2.80 \mathrm{~m}$, surface alt $148.3 \mathrm{~m}$, Kilpisuo bog $\left(62^{\circ} 25^{\prime} \mathrm{N}, 26^{\circ} 09^{\prime} \mathrm{E}\right)$. Coll 1973 by A Leino and subm 1973 by V E Valovirta.

\section{Su-302. Äänekoski, Central Finland}

$$
\begin{array}{r}
\mathbf{8 3 0 0} \pm \mathbf{1 0 0} \\
\delta^{13} \mathrm{C}=-25.9 \%
\end{array}
$$

From pine wood above sand, taken with piston sampler, depth 6.77 to $6.80 \mathrm{~m}$, surface alt $113.8 \mathrm{~m}$, Kaikkarsuo bog $\left(62^{\circ} 39^{\prime} \mathrm{N}, 25^{\circ} 30^{\prime} \mathrm{E}\right)$. Coll 1973 by A Leino and subm 1973 by V E Valovirta.

\section{Su-308. Äänekoski, Central Finland}

$$
\begin{array}{r}
\mathbf{7 3 8 0} \pm \mathbf{8 0} \\
\delta^{18} C=-29.2 \% \text { o }
\end{array}
$$

Same site as Su-302, peat, depth 6.77 to $6.80 \mathrm{~m}$, taken with piston sampler. Coll 1973 by A Leino and subm by V E Valovirta.

\section{Su-303. Ä̈̈nekoski, Central Finland}

$4390 \pm 60$

$\delta^{13} C=-29.3 \%$ o

Shore peat above silt, taken with piston sampler, depth 3.12 to $3.16 \mathrm{~m}$, surface alt $89 \mathrm{~m}$, Orissuo bog $\left(62^{\circ} 30^{\prime} \mathrm{N}, 26^{\circ} 03^{\prime} \mathrm{E}\right)$. Coll 1973 by $\mathrm{A}$ Leino and subm 1973 by V E Valovirta.

\section{Su-304. Äänekoski, Central Finland}

$$
\begin{array}{r}
\mathbf{8 9 8 0} \pm \mathbf{1 0 0} \\
\delta^{13} C=-29.0 \%
\end{array}
$$

Peat above silt, taken with piston sampler, depth 4.16 to $4.20 \mathrm{~m}$, surface alt $178.4 \mathrm{~m}$, Isosuo bog $\left(62^{\circ} 34^{\prime} \mathrm{N}, 25^{\circ} 31^{\prime} \mathrm{E}\right)$. Coll 1973 by $\mathrm{A}$ Leino and subm 1973 by V E Valovirta. 
Su-305. Perniö, S Finland

$5960 \pm 90$

$\delta^{13} \mathrm{C}=-26.5 \%$

Wood and peat above gyttja (detritus) taken with piston sampler, depth 3.97 to $4 \mathrm{~m}$, surface alt $43 \mathrm{~m}$ (threshold), Melassuo bog $\left(60^{\circ} 12^{\prime} \mathrm{N}\right.$, $23^{\circ} 09^{\prime} \mathrm{E}$ ). Coll 1973 by A Leino and subm 1973 by V E Valovirta.

\section{Su-306. Perniö, S Finland}

$6290 \pm 80$

Peat and wood above sand, taken wit to $4.65 \mathrm{~m}$, surface alt $40.5 \mathrm{~m}$, Träskmossan Coll 1973 by A Leino and subm by V E Valog (60 $\left.05^{\prime} \mathrm{N}, 23^{\circ} 02^{\prime} \mathrm{E}\right)$.

\section{Su-307. Perniö, S Finland}

$$
\begin{array}{r}
\mathbf{6 2 9 0} \pm \mathbf{6 0} \\
\delta^{13} \mathrm{C}=-29.2 \%
\end{array}
$$

Peat taken with piston sampler, depth 3.71 to $3.75 \mathrm{~m}$, surface alt $43.15 \mathrm{~m}$, Lakiassuo bog $\left(60^{\circ} 12^{\prime} \mathrm{N}, 23^{\circ} 57^{\prime} \mathrm{E}\right)$. Coll 1973 by A Leino and subm by V E Valovirta.

\section{Su-311. Sodankylä, N Finland}

$$
\begin{aligned}
& >\mathbf{5 5 , 0 0 0} \\
\delta^{13} C & =-26.0 \% \%
\end{aligned}
$$

Peat $(\mathrm{CB})$ taken with piston sampler, depth 3.6 to $3.8 \mathrm{~m}$, surface alt $347 \mathrm{~m}$, Pyssyselkä $\left(67^{\circ} 53^{\prime} \mathrm{N}, 26^{\circ} 08^{\prime} \mathrm{E}\right)$. Coll and subm 1973 by $\mathrm{H}$ Tanskanen (5 2-day counts) (Tanskanen, 1975).

\section{Su-312. Sodankylä, N Finland}

Same site as Su-311. Peat (CB), depth 3.8 to $4.05 \mathrm{~m}$. Coll and subm 1973 by H Tanskanen (4 2-day counts) (Tanskanen, 1975).

\section{Su-313. Inari, N Finland}

Wood from peat layer between sand in erosion bank of river Ivalojoki Törmänen $\left(68^{\circ} 36.7^{\prime} \mathrm{N}, 27^{\circ} 28.6^{\prime} \mathrm{E}\right)$, surface alt $122 \mathrm{~m}$, depth $1.7 \mathrm{~m}$. Coll and subm 1973 by A T Lahtinen. Comment (ATL): Analysis of Su-314 was made of peat layer mentioned below. Possibly drift wood transported by flood.

\section{Su-314. Inari, N Finland}

$$
\begin{array}{r}
1270 \pm 90 \\
\delta^{13} C=-24.2 \%
\end{array}
$$

Peat between sand layers in erosion bank of Ivalojoki at village of Törmänen. Sample was taken from peat layer mentioned in Su-313 at depth of 1.65 to $1.75 \mathrm{~m}$. Coll and subm 1973 by A T Lahtinen. Comment (ATL): peat was formed when old meander channel grew into a bog, after which river action buried peat layer under ca $1.5 \mathrm{~m}$ alluvial sand. Thickness of peat layer varies from 10 to $30 \mathrm{~cm}$.

\section{Su-315. Inari, N Finland}

$$
\begin{array}{r}
970 \pm 60 \\
\delta^{13} C=-24.5 \% 。
\end{array}
$$

Peat under sand from hand-dug section, near village of Akujärvi $\left(68^{\circ} 40.9^{\prime} \mathrm{N}, 27^{\circ} 39.4^{\prime} \mathrm{E}\right)$, surface alt $122 \mathrm{~m}$, depth 0.55 to $0.63 \mathrm{~m}$. Coll and subm 1973 by A T Lahtinen. Comment (ATL): peat layer ca $20 \mathrm{~cm}$ 
thick was formed when delta channel grew into a bog, after which it was buried under ca $0.5 \mathrm{~m}$ sand.

Su-316. Multia, Central Finland

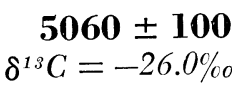

Subfossil hazelnuts (Corylus avellana) from hand-dug sec, depth 0.8 to $1.1 \mathrm{~m}$, village of Isojärvi, Heposuo bog $\left(62^{\circ} 20^{\prime} \mathrm{N}, 24^{\circ} 57^{\prime} \mathrm{E}\right)$. Coll 1973 by L O Ervi and subm 1973 by V E Valovirta.

\section{Su-317. Multia, Central Finland}

$$
\begin{array}{r}
4985 \pm 100 \\
\delta^{13} C=-22.4 \% \text { \% }
\end{array}
$$

Subfossil hazelnuts (Corylus avellana) from hand-dug sec of bog earth pit, depth 0.8 to $1.1 \mathrm{~m}$, village of Sahrajärvi, Uusi Ilomäki $\left(62^{\circ} 28^{\prime}\right.$ N, 25 $05^{\prime}$ E). Coll 1973 by L O Ervi and subm 1973 by V E Valovirta.

\section{Jukolansuo bog series, Kytäjä, S Finland}

Peat, gyttja and silt samples from hand-dug sec, surface alt $121 \mathrm{~m}$, Jukolansuo bog $\left(60^{\circ} 35^{\prime} \mathrm{N}, 24^{\circ} 36^{\prime} \mathrm{E}\right)$. Coll 1973 by A Leino and V E Valovirta and subm 1974 by V E Valovirta ( $c f$ Heikkinen 1971, p 434).

\section{Su-377. Jukolansuo}

Modern $\Delta^{14} \mathbf{C}=+\mathbf{4 3} \%$ o $\delta^{13} C=-26.4 \%$ o

Peat, depth 0.08 to $0.1 \mathrm{~m}$. Comment: pollen analysis shows end of Sub-Atlantic period and rise of Picea.

\section{Su-378. Jukolansuo}

$110 \pm 40$

$\delta^{13} C=-25.5 \%$ o

Peat, depth 0.18 to $0.2 \mathrm{~m}$. Comment: pollen analysis shows SubAtlantic period and Picea minimum.

\section{Su-379. Jukolansuo}

$$
\begin{array}{r}
\mathbf{8 7 0} \pm \mathbf{3 0} \\
\delta^{13} C=-25.8 \% \text { 。 }
\end{array}
$$

Peat, depth 0.28 to $0.3 \mathrm{~m}$. Comment: pollen analysis shows SubAtlantic period and Picea minimum.

\section{Su-380. Jukolansuo}

$$
\begin{array}{r}
\mathbf{1 3 3 0} \pm \mathbf{5 0} \\
\delta^{13} C=-26.1 \%
\end{array}
$$

Peat, depth 0.38 to $0.4 \mathrm{~m}$. Comment: pollen analysis shows SubAtlantic period.

\section{Su-381. Jukolansuo}

$$
\begin{array}{r}
\mathbf{2 0 5 0} \pm \mathbf{4 0} \\
\delta^{13} C=-25.8 \% \text {. }
\end{array}
$$

Peat, depth 0.48 to $0.5 \mathrm{~m}$. Comment: pollen analysis shows SubAtlantic period.

\section{Su-382. Jukolansuo}

$$
\begin{array}{r}
\mathbf{2 7 4 0} \pm \mathbf{4 0} \\
\delta^{1 s} C=-26.7 \%
\end{array}
$$

Peat, depth 0.58 to $0.6 \mathrm{~m}$. Comment: pollen analysis shows rise of Picea pollen and transition from Sub-Boreal to Sub-Atlantic period. 
Su-383. Jukolansuo

$$
\begin{array}{r}
\mathbf{5 2 5 0} \pm \mathbf{5 0} \\
\delta^{13} C=-26.7 \% \text { o }
\end{array}
$$

Peat, depth 0.68 to $0.7 \mathrm{~m}$. Comment: pollen analysis shows end of Atlantic period.

\section{Su-384. Jukolansuo}

$$
\begin{array}{r}
6520 \pm 100 \\
\delta^{13} C=-26.7 \% \text { o }
\end{array}
$$

Wood peat, depth 0.78 to $0.8 \mathrm{~m}$. Comment: pollen analysis shows Atlantic period.

\section{Su-385. Jukolansuo}

$$
6740 \pm 40
$$

Wood peat, depth 0.88 to $0.9 \mathrm{~m}$. Comment: pollen Atlantic period.

Su-386. Jukolansuo

Wood peat, depth 0.98 to $1 \mathrm{~m}$. Comment: pollen analysis shows Atlantic period.

\section{Su-387. Jukolansuo}

$$
7460 \pm 80
$$
period.

Peat, depth 1.08 to $1.1 \mathrm{~m}$. Comment: pollen analysis shows Atlantic

\section{Su-388. Jukolansuo}

$$
7900 \pm 100
$$

Peat, depth 1.18 to $1.2 \mathrm{~m}$. Comment: pollen analysis shows end of Boreal period.

\section{Su-389. Jukolansuo}

Peat, depth 1.28 to $1.3 \mathrm{~m}$. Comment: pollen analysis shows transition from Pre-Boreal to Boreal period.

\section{Su-390. Jukolansuo}

Gyttja (detritus), depth 1.38 to $1.4 \mathrm{~m}$. Comment: pollen analys shows Pre-Boreal period.

\section{Su-391. Jukolansuo}

Gyttja (detritus), depth 1.48 to $1.5 \mathrm{~m}$. Co shows Pre-Boreal period.

\section{Su-392. Jukolansuo}

Gyttja silt, depth 1.58 to $1.6 \mathrm{~m}$. Comment: pollen analysis $\mathbf{9 4 9 0 \pm 1 4 0}$ Boreal period.

\section{Su-393. Lammi, S Finland}

$8810 \pm 100$

Clay gyttja with moss remnants (mostly Drepanocladus fluitans), "Silmisuo" (NW part of Kaurastensuo bog) (61 $\left.02^{\circ} \mathrm{N}, 24^{\circ} 59^{\prime} \mathrm{E}\right)$, surface alt $152 \mathrm{~m}$, depth 5.78 to $5.82 \mathrm{~m}$. Coll 1973 and subm 1974 by $\mathrm{K}$ Tolonen. Comment (KT): according to pollen, diatom and cladoceran 
analyses sample represents the 1st organic sedimentation after deglaciation. If result is compared with Su-394 and with the standard pollen diagrams by Tolonen \& Ruuhijärvi (1976), age seems to be ca $1000 \mathrm{yr}$ "too young".

\section{Su-394. Lammi, S Finland}

$9040 \pm 80$

Same site as Su-393. Gyttja (detritus) at depth 5.67 to $5.68 \mathrm{~m}$. Coll 1973 and subm 1974 by K Tolonen. Comment: sample represents transition Betula/Pinus in Lammi area. Result consistent with radiocarbon chronology of pollen zones in Lammi area (Tolonen \& Ruuhijärvi, 1976).

\section{Su-395. Vaala, N Finland}

$8120 \pm 80$

Peat (BC) overlying gyttja (detritus), depth 3.85 to $3.9 \mathrm{~m}$, surface alt $122.5 \mathrm{~m}$, Oulujärvi $\left(64^{\circ} 27^{\prime} \mathrm{N}, 27^{\circ} 10^{\prime} \mathrm{E}\right)$. Coll 1974 with piston sampler and subm 1974 by J Häikiö. Comment: pollen analysis shows transition from Boreal to Atlantic period.

\section{Bastuberg series, Porvoo, S Finland}

Peat and gyttja, taken with piston sampler, Bastuberg bog $\left(60^{\circ} 21^{\prime}\right.$ $\mathrm{N}, 25^{\circ} 47^{\prime} \mathrm{E}$ ), surface alt $28.5 \mathrm{~m}$. Coll 1974 by A Leino and V E Valovirta and subm 1975 by V E Valovirta.

Su-474. Bastuberg, Core A

$120 \pm 120$

Peat (LSC), depth 0.2 to $0.24 \mathrm{~m}$.

Su-475. Bastuberg, Core A

$1160 \pm 60$

Peat (LSC), depth 0.4 to $0.44 \mathrm{~m}$.

Su-476. Bastuberg, Core A

$5180 \pm 65$

Peat (LSC), depth 0.8 to $0.84 \mathrm{~m}$. Comment: pollen analysis shows Atlantic period.

Su-477. Bastuberg, Core A

$\mathbf{5 8 9 0} \pm \mathbf{7 0}$

Gyttja (detritus), depth 1 to $1.04 \mathrm{~m}$. Comment: pollen analysis shows Atlantic period.

Su-478. Bastuberg, Core A

$\mathbf{7 4 4 0} \pm 90$

Gyttja (detritus), depth 1.45 to $1.49 \mathrm{~m}$. Comment: pollen analysis shows beginning of Atlantic period.

Su-479. Bastuberg, Core A

$7850 \pm 90$

Gyttja (detritus), depth 1.96 to $2 \mathrm{~m}$. Comment: pollen analysis shows beginning of Atlantic period.

Su-480. Bastuberg, Core A

$8480 \pm 100$

Gyttja (detritus), depth 2.2 to $2.25 \mathrm{~m}$. Comment: pollen analysis shows Boreal period. 
Su-481. Bastuberg, Core A

$9150 \pm 200$

Gyttja (detritus), depth 2.4 to $2.45 \mathrm{~m}$. Comment: pollen analysis shows Pre-Boreal period.

Su-482. Bastuberg, Core $B$

Peat (S), depth 0.18 to $0.22 \mathrm{~m}$.

Su-483. Bastuberg, Core B

Peat (S), depth 0.38 to $0.42 \mathrm{~m}$.

\section{Su-484. Bastuberg, Core B}

Peat (LS), depth 0.78 to $0.82 \mathrm{~m}$. Comment: pollen analysis shows Sub-Boreal period.

\section{Su-485. Bastuberg, Core B}

$5350 \pm 100$

Peat (LS), depth 0.98 to $1.02 \mathrm{~m}$. Comment: pollen analysis shows transition from Atlantic to Sub-Boreal period.

\section{Kerkkolankangas series, Jämsänkoski, Central Finland}

Till samples containing organic matter from gravel pit at Kerkkolankangas $\left(61^{\circ} 55^{\prime} \mathrm{N}, 25^{\circ} 09^{\prime} \mathrm{E}\right)$. Till bed is in proximal part of a marginal formation, between glaciofluvial deposits, at depth ca 6 to $7 \mathrm{~m}$. Surface alt $140 \mathrm{~m}$. Coll 1974 with spade by T Ruohomäki and M Putkinen and subm 1975 by P Lahermo and H Rainio (Rainio \& Lahermo, 1976).

\section{Su-492. Kerkkolankangas $B$}

Sample from middle layer of 3-layer till bed. Comment: analysis based on humic acids, sample $9 \mathrm{~kg}, 0.5 \mathrm{~N} \mathrm{NaOH}$ leach, HCl precipitation (2 2-day counts).

\section{Su-504. Kerkkolankangas B}

$>45,000$

Same site as Su-492. Comment: analysis from $3 \mathrm{~kg}$ till without chemical treatment (2 2-day counts).

\section{Su-493. Kerkkolankangas $\mathrm{C}$}

Sample from lowermost layer of 3-layer till bed. Comment: analysis based on humic acids, sample 19.2kg (2 2-day counts).

\section{Honkalanmäki series, Kuru, Central Finland}

Till samples containing organic matter from road-cut at Honkalanmäki $\left(61^{\circ} 45^{\prime} \mathrm{N}, 26^{\circ} 36^{\prime} \mathrm{E}\right)$ from basal till on lee side of rock hummock overlain by sand, silt and till $4 \mathrm{~m}$ thick. Surface alt $120 \mathrm{~m}$. Coll 1974 with spade by T Ruohomäki and M Putkinen and subm 1975 by P Lahermo and H Rainio (Rainio \& Lahermo, 1976). 
Su-494. Honkalanmäki B

Sample from topmost layer of 3-layer till bed. Comment: analysis from humic acids, sample $18.6 \mathrm{~kg}, 0.5 \mathrm{~N} \mathrm{NaOH}$ leach, $\mathrm{HCl}$ precipitation (2 2-day counts).

Su-495. Honkalanmäki C

Sample from middle layer of 3-layer till bed. Comment: analysis from humic acids, sample $18 \mathrm{~kg}$ (2 2-day counts).

\section{Osuuspankki series, Kuopio, Central Finland}

Samples from ancient bog covered by sand deposits, from foundation pit excavated for Osuuspankki bldg $\left(62^{\circ} 53.5^{\prime} \mathrm{N}, 27^{\circ} 41^{\prime} \mathrm{E}\right)$. Coll 1975 by L Timgren and subm 1975 by V E Valovirta (Kotilainen, 1953).

\section{Su-496. Osuuspankki, Sample 1}

$7760 \pm 100$

Peat from lowermost part of peat, $0.4 \mathrm{~m}$ thick, between 2 sand beds, depth $3.1 \mathrm{~m}$, surface alt $95.6 \mathrm{~m}$.

Su-497. Osuuspankki, Sample 3

$8170 \pm 100$

Wood, same site as Su- 496 .

Su-498. Osuuspankki, Sample 4

$700 \pm 100$

Mud and peat, depth $1 \mathrm{~m}$, surface alt $97.7 \mathrm{~m}$.

\section{Kurujoki series, Sodankylä, N Finland}

Samples from various levels of till deposit covered by postglacial peat bog near Kurujoki brook $\left(67^{\circ} 58^{\prime} \mathrm{N}, 27^{\circ} 18^{\prime} \mathrm{E}\right)$, surface alt $244 \mathrm{~m}$. Coll with piston sampler (and jackhammer piston sampler) 1975 by $\mathrm{E}$ Magga and subm 1975 by R Kujansuu and S Leskelä.

\section{Su-507. Kurujoki}

$9060 \pm 100$

Peat from bottom of postglacial peat layer at depth of 1.45 to $1.52 \mathrm{~m}$.

\section{Su-508. Kurujoki}

$>\mathbf{5 0 , 0 0 0}$

Peat layer sandwiched in flood-plain sediments overlain by till and postglacial peat, depth 4.3 to $4.5 \mathrm{~m}$ (Hirvas et al, 1976) (5 2-day counts).

\section{Su-509. Kurujoki}

$>\mathbf{5 0 , 0 0 0}$

Peat layer sandwiched in flood-plain sediments overlain by till and postglacial peat, depth 6.86 to $7.78 \mathrm{~m}$ (Hirvas et al, 1976) (3 2-day counts). Su-510. Kittilä, N Finland

Peat overlain by till, depth 5.93 to $6.50 \mathrm{~m}$, surface alt ca $200 \mathrm{~m}$, Naakenavaara $\left(67^{\circ} 42^{\prime} \mathrm{N}, 24^{\circ} 7.1^{\prime} \mathrm{E}\right)$. Coll 1975 with jackhammer piston sampler by E Magga and subm 1975 by R Kujansuu and S Leskelä (4 2-day counts).

\section{Su-511. Sodankylä, N Finland}

$7140 \pm 120$

Peat from bottom of postglacial peat layer, depth 2.7 to $2.9 \mathrm{~m}$, surface alt ca $208 \mathrm{~m}$, Ilmakkiselkä $\left(67^{\circ} 43^{\prime} \mathrm{N}, 26^{\circ} 38^{\prime} \mathrm{E}\right)$. Coll 1975 with jack- 
hammer piston sampler by E Magga and subm 1975 by R Kujansuu and S Leskelä.

\section{Su-512. Sodankylä, N Finland}

$$
9130 \pm 150
$$

Peat from bottom of postglacial peat layer, depth 3.8 to $4.02 \mathrm{~m}$, surface alt ca $207 \mathrm{~m}$, Postoaapa $\left(67^{\circ} 38.5^{\prime} \mathrm{N}, 26^{\circ} 36.4 \mathrm{E}\right)$. Coll 1975 with piston sampler by E Magga and subm 1975 by $\mathrm{R}$ Kujansuu and $\mathrm{S}$ Leskelä.

\section{Su-513. Sodankylä, N Finland}

Same site as Su-512. Gyttja overlain by till deposits, depth 6 to $6.5 \mathrm{~m}$. Coll with jackhammer piston sampler by E Magga and subm 1975 by R Kujansuu and S Leskelä (4 2-day counts).

\section{Su-514. Kristiinankaupunki, W Finland}

Till-covered silty sand with root remnants in gravel pit, depth $1.5 \mathrm{~m}$, surface alt $57 \mathrm{~m}$, Risåsen $\left(62^{\circ} 12.5^{\prime} \mathrm{N}, 21^{\circ} 38^{\prime} \mathrm{E}\right)$. Coll and subm 1975 by J Niemelä and R Tynni.

\section{Mieslahti series, Paltamo, E Finland}

Samples from various levels in bog near Mieslahti $\left(64^{\circ} 22^{\prime} \mathrm{N}, 28^{\circ}\right.$ $00^{\prime} \mathrm{E}$ ), surface alt $122.5 \mathrm{~m}$. Coll 1975 with piston sampler and subm 1975 by J Häikiö.

\section{Su-515. Mieslahti}

Peat (EqC) from immediately below silty gyttja, depth 4.05 to $4.1 \mathrm{~m}$. Bog was flooded by water of Lake Oulujärvi.

\section{Su-516. Mieslahti}

$$
6430 \pm 60
$$

Peat (EqSC) overlying silty gyttja, depth 5.4 to $5.5 \mathrm{~m}$. Comment: end of flood or transgression that caused silt layer.

\section{Su-517. Mieslahti}

Peat (EqG) mixed with detritus underlying gyttja and silt, depth 6 to $6.1 \mathrm{~m}$. Comment: pollen analysis shows transition from Boreal to Atlantic period.

\section{Su-541. Kristiinankaupunki, W Finland}

Sand containing charcoal in gravel pit, depth $1.5 \mathrm{~m}$, surface alt $57 \mathrm{~m}$, Risåsen $\left(62^{\circ} 12.5^{\prime} \mathrm{N}, 21^{\circ} 38^{\prime} \mathrm{E}\right)$. Coll 1975 by R Tynni and K Hokkanen and subm 1975 by J Niemelä and R Tynni (1975). Comment: stratigraphic and microfossil determinations indicate that carboniferous layer was deposited during previous interstadial or interglacial period. $C f$ Su-573 (2 2-day counts).

\section{Su-573. Kristiinankaupunki, W Finland}

Till-covered esker, charcoal in sand in gravel pit, depth $3 \mathrm{~m}$, surface alt $45 \mathrm{~m}$, Risåsen $\left(62^{\circ} 13^{\prime} \mathrm{N}, 21^{\circ} 37.8^{\prime} \mathrm{E}\right), 600 \mathrm{~m} \mathrm{~N}$ from site of Su-541. Coll by R Tynni and K Hokkanen and subm 1975 by J Niemelä and R Tynni (4 2-day counts). 
Su-542. Vaala, N Finland

$\mathbf{3 0 0} \pm \mathbf{3 0}$

Stump, exposed from below a beach ridge, surface alt $122.5 \mathrm{~m}$, Kuostonsaari $\left(64^{\circ} 27^{\prime} \mathrm{N}, 27^{\circ} 10^{\prime} \mathrm{E}\right)$. Coll and subm 1975 by J Häikiö.

\section{Sammaljoensuo bog series, Ylitornio, N Finland}

Samples from various sites and levels in Sammaljoensuo bog $\left(66^{\circ}\right.$ $\left.26^{\prime} \mathrm{N}, 23^{\circ} 5 \mathrm{l}^{\prime} \mathrm{E}\right)$, surface alt $105 \mathrm{~m}$. Coll 1975 with piston sampler by P Lahermo and V E Valovirta (Lahermo et al, 1977).

Su-543. Sammaljoensuo, C-D, 105m

$6230 \pm 60$ fication.

Wood, depth 3.72 to $3.83 \mathrm{~m}$. Comment: dates beginning of paludi-

Su-544. Sammaljoensuo, C-D, 205m

$6830 \pm 120$

Peat (LSB), depth 4.2 to $4.24 \mathrm{~m}$. Comment: dates beginning of paludification.

Su-545. Sammaljoensuo, A-B, 105m $7150 \pm 50$

Peat (LSB), depth 4.35 to $4.41 \mathrm{~m}$. Comment: pollen analysis shows beginning of Atlantic period.

Su-546. Sammaljoensuo, A-B, 105m $7900 \pm 150$

Peat (LSB), depth 4.58 to $4.64 \mathrm{~m}$. Comment: pollen analysis shows Boreal period.

\section{Värttiövaara series, Kittilä, N Finland}

Samples from various sites and levels in Värttiövaara bog $\left(67^{\circ} 33^{\prime} \mathrm{N}\right.$, $25^{\circ} 45^{\prime} \mathrm{E}$ ), surface alt $214 \mathrm{~m}$. Coll 1975 with piston sampler by $\mathrm{P}$ Lahermo, A Leino and M Putkinen and subm 1975 by P Lahermo and V E Valovirta (Lahermo et al, 1977).

Su-547. Värttiövaara, A-B, Om $4660 \pm 40$

Peat (BSC), depth 0.95 to $1 \mathrm{~m}$. Comment: pollen analysis shows SubBoreal period.

Su-548. Värttiövaara, A-B, $7 \mathrm{~m}$ $\mathbf{8 8 1 0} \pm \mathbf{5 0}$

Peat (BSC), depth 1.9 to $1.95 \mathrm{~m}$. Comment: pollen analysis shows Pre-Boreal period.

\section{Säynäjäjärvi series, Kittilä, N Finland}

Samples from various sites and levels in Säynäjäjärvi bog $\left(67^{\circ} 21^{\prime} \mathrm{N}\right.$, $25^{\circ} 24^{\prime} \mathrm{E}$ ), surface alt $215 \mathrm{~m}$. Coll 1975 with piston sampler by $\mathrm{P}$ Lahermo, $\mathrm{A}$ Leino and M Putkinen and subm 1975 by $\mathrm{P}$ Lahermo and V E Valovirta (Lahermo et al, 1977).

Su-549. Säynäjäjärvi, A-B, 20m $4530 \pm 40$

Peat (BS), depth 1.95 to $2 \mathrm{~m}$. Comment: pollen analysis shows Atlantic period. 
Su-550. Säynäjäjärvi, A, $32 \mathrm{~m}$

$$
7920 \pm 70
$$

Peat (BS), depth 2.76 to $2.8 \mathrm{~m}$. Comment: pollen analysis shows beginning of Atlantic period.

\section{Tuorenaakiselkä series, Sodankylä, N Finland}

Samples from various levels and sites in Tuorenaakiselkä bog $\left(67^{\circ}\right.$ $46^{\prime} \mathrm{N}, 25^{\circ} 58^{\prime} \mathrm{E}$ ), surface alt $296 \mathrm{~m}$. Coll 1975 with piston sampler by $\mathrm{P}$ Lahermo, A Leino and M Putkinen and subm 1975 by P Lahermo and V E Valovirta (Lahermo et al, 1977).

Su-551. Tuorenakiselkä, A-B, $10 \mathrm{~m}$

$$
8640 \pm 120
$$

Peat (LSB), depth 2.06 to $2.11 \mathrm{~m}$. Comment: pollen analysis shows Pre-Boreal period.

Su-552. Tuorenakiselkä, C-D, $14 \mathrm{~m}$

$7910 \pm 60$

Peat (LSB), depth 1.95 to $2 \mathrm{~m}$. Comment: pollen analysis shows beginning of Atlantic period.

Su-553. Tuorenaakiselkä, C-D, 29m

Peat (CB), depth 276 to $2.8 \mathrm{~m} . \mathrm{Comm}, \mathbf{8 7 9 0} \pm \mathbf{7 0}$ Boreal period.

\section{Hattuvaara series, Suomussalmi, E Finland}

Samples from various levels of Hattuvaara bog $\left(65^{\circ} 06^{\prime} \mathrm{N}, 28^{\circ} 39^{\prime} \mathrm{E}\right)$, surface alt $262 \mathrm{~m}$. Coll 1975 with piston sampler by A Leino and subm 1975 by V E Valovirta.

\section{Su-556. Hattuvaara}

$$
160 \pm 50
$$

Peat (S), depth 0.2 to $0.23 \mathrm{~m}$. Comment: pollen analysis shows SubAtlantic period.

\section{Su-557. Hattuvaara}

Peat (ErS), depth 0.4 to $0.43 \mathrm{~m}$. Comment: pollen analysis shows SubBoreal period.

\section{Su-558. Hattuvaara}

$3640 \pm 60$

Peat (ErS), depth 0.6 to $0.63 \mathrm{~m}$. Comment: pollen analysis shows SubBoreal period.

\section{Su-559. Hattuvaara}

Peat (CS), depth 0.8 to $0.83 \mathrm{~m}$. Comment: rise of Picea pollen curve starts at this level.

\section{Su-560. Hattuvaara}

Peat (CS), depth 1 to $1.03 \mathrm{~m}$. Comment: pollen analysis shows transition from Atlantic to Sub-Boreal period.

\section{Su-561. Hattuvaara}

Peat (SC), depth 1.2 to $1.23 \mathrm{~m}$. Comment: pollen analysis shows Atlantic period. 
Su-562. Hattuvaara

$6920 \pm 90$

Peat (SC), depth 1.4 to $1.43 \mathrm{~m}$. Comment: pollen analysis shows Atlantic period.

Su-563. Hattuvaara

$7010 \pm 110$

Peat (SC), depth 1.6 to $1.63 \mathrm{~m}$. Comment: pollen analysis shows Atlantic period.

Su-564. Hattuvaara

$7210 \pm 80$

Peat (SC), depth 1.7 to $1.72 \mathrm{~m}$. Comment: pollen analysis shows Atlantic period.

Su-565. Hattuvaara

$7590 \pm 90$

Peat (SC), depth 1.8 to $1.82 \mathrm{~m}$. Comment: pollen analysis shows transition from Boreal to Atlantic period.

Su-566. Hattuvaara

$7900 \pm 50$

Peat (BC), depth 1.89 to $1.92 \mathrm{~m}$. Comment: pollen analysis shows end of Boreal period.

Su-567. Hattuvaara

$8040 \pm 80$

Peat (EqC), depth 1.94 to $1.97 \mathrm{~m}$. Comment: pollen analysis shows Boreal period.

\section{Su-568. Hattuvaara}

$8380 \pm 90$ period.

Gyttja, depth 2.02 to $2.05 \mathrm{~m}$. Comment: pollen analysis shows Boreal

\section{Su-569. Hattuvaara}

$$
8790 \pm 90
$$

Gyttja, depth 2.1 to $2.13 \mathrm{~m}$. Comment: pollen analysis shows beginning of Boreal period.

\section{Su-570. Hattuvaara}

$8810 \pm 150$

Gyttja, depth 2.18 to $2.21 \mathrm{~m}$. Comment: pollen analysis shows transition from Pre-Boreal to Boreal period.

\section{Su-571. Hattuvaara}

$8830 \pm 140$

Gyttja, depth 2.26 to $2.27 \mathrm{~m}$. Comment: pollen analysis shows end of Pre-Boreal period.

\section{Su-572. Kuopio, Central Finland}

$6250 \pm 100$

Wood and peat sample from peat layer in sand, Savilahti $\left(62^{\circ} 54.5^{\prime}\right.$ $\mathrm{N}, 27^{\circ} 37.8^{\prime} \mathrm{E}$ ), surface alt $90 \mathrm{~m}$, depth $3 \mathrm{~m}$. Coll 1975 by $\mathrm{H}$ Rainio and T Ruohomäki and subm 1975 by H Rainio.

\section{Su-574. Bothnian Sea}

$$
6600 \pm 180
$$

Gyttja-banded clay, Bothnian Sea $\left(61^{\circ} 40^{\prime} \mathrm{N}, 20^{\circ} 50^{\prime} \mathrm{E}\right)$, depth ca $3 \mathrm{~m}$, water depth ca $85 \mathrm{~m}$, sample taken 0 to $5 \mathrm{~cm}$ above Litorina/Ancylus boundary. Coll 1974 with piston corer by E Kukkonen and subm 1975 by $\mathrm{H}$ Ignatius. Comment: sample subjected to non-chemical processing. 


\section{Su-575. Bothnian Sea}

Homogeneous clay containing some organic matter, same site as Su-574, sample taken 0 to $10 \mathrm{~cm}$ below Litorina/Ancylus boundary. Coll 1974 with piston corer by E Kukkonen and subm 1975 by H Ignatius. Comment: sample subjected to non-chemical processing.

\section{ARCHAEOLOGIC SAMPLES}

\section{Finland}

\section{Early agricultural history in SW Finland}

Su-428. Tenhola, SW Finland

$1240 \pm 50$

Dark humus-rich gyttja clay, Bonästräsket $\left(60^{\circ} 03^{\prime} \mathrm{N}, 23^{\circ} 22^{\prime} \mathrm{E}\right)$, surface alt $1 \mathrm{~m}$, depth 1 to $1.05 \mathrm{~m}$, water depth ca $10 \mathrm{~m}$. Coll 1974 by K Tolonen and A Siiriäinen and subm 1974 by $\mathrm{K}$ Tolonen. Comment: according to land-uplift chronology from diatoms, horizon would date to ca $\mathrm{AD} 1400$, ie, radiocarbon age is ca $700 \mathrm{yr}$ "too old". Old fossil organic carbon in sediment obviously originates from "field erosion" in catchment area of basin owing to beginning of intensive rye cultivation (Tolonen \& Ruuhijärvi, 1976; Tolonen, Siiriäinen \& Hirviluoto, 1977).

\section{Läppträsket series, Karjaa, SW Finland}

Samples from various sites and levels, Lake Läppträsket $\left(60^{\circ} 03^{\prime} \mathrm{N}\right.$, $23^{\circ} 44^{\prime} \mathrm{E}$ ), surface alt $5.2 \mathrm{~m}$. Coll 1974 by A Siiriäinen and K Tolonen and subm 1974 by K Tolonen.

General Comment: results from Läppträsket cores agree with each other and with land-uplift chronology as well as with corresponding dates obtained from Espoo area (Tolonen et al, 1975a;b).

\section{Su-429. Läppträsket, Core A}

Fine detritus gyttja, depth 2.55 to $2.65 \mathrm{~m}$. Comment: horizon represents 1st occurrence of rye in pollen diagram (Tolonen \& Ruuhijärvi, 1976; Tolonen, Siiriäinen \& Hirviluoto, 1977).

\section{Su-430. Läppträsket, Core $A$}

$940 \pm 100$

Dark coarse detritus gyttja from 1.8 to $1.85 \mathrm{~m}$. Comment: start of "upper occurrence" of wheat and rye (op cit, above).

\section{Su-432. Läppträsket, Core D}

Dark organic gyttja clay from 2.45 to $2.55 \mathrm{~m}$. Comment: 1 st occurrence of rye in pollen diagram at end of brackish water stage (op cit, above).

\section{Su-433. Läppträsket, Core D}

$$
1030 \pm 50
$$

Dark coarse detritus gyttja from 1.85 to $1.95 \mathrm{~m}$. Comment: upper occurrence of cereals after gap ca AD 600 to 900. Result agrees with history of settlement (op cit, above). 
Sphagnum fuscum peat from hand-dug sec from raised bog Lintunemossen $\left(63^{\circ} 07^{\prime} 30^{\prime \prime} \mathrm{N}, 22^{\circ} 10^{\prime} \mathrm{E}\right)$, surface alt $17.5 \mathrm{~m}$, depth 0.8 to $0.82 \mathrm{~m}$. Coll 1973 by A Siiriäinen and K Tolonen and subm 1974 by $\mathrm{K}$ Tolonen. Comment: "upper occurrence" of cultural pollen grains and cereals. Age agrees with growth-rate curve of bog obtained by means of isolation niveau at depth $1.6 \mathrm{~m}$, ca $1800 \mathrm{BP}$, dating Hel-705: 750 \pm 100 $\mathrm{BP}$, at depth 0.6 to $0.63 \mathrm{~m}$, and natural bog surface (op cit, above).

\section{Su-435. Laitila "II", SW Finland $\quad \mathbf{1 2 1 0} \pm \mathbf{5 0}$}

Forest peat from hand-dug sec from raised bog Isorahka $\left(60^{\circ} 54^{\prime} \mathrm{N}\right.$, $\left.21^{\circ} 38^{\prime} \mathrm{E}\right)$, surface alt $15 \mathrm{~m}$, depth from 1.61 to $1.71 \mathrm{~m}$. Coll 1973 and subm 1974 by K Tolonen. Comment: date agrees with other dates from same profile as well as those from beginning of rye pollen curve in latter half of Iron age in Laitila area (op cit, above).

\section{Su-436. Laitila "II", SW Finland}

Forest peat from same place as $\mathrm{Su}-435$, depth from 2.4 to $2.49 \mathrm{~m}$. Comment: date agrees with land uplift chronology as well as with other dates from beginning of cerealia curve in Early Roman Iron age in Laitila area (op cit, above).

\section{Su-437. Laitila "IX", SW Finland}

$2310 \pm 50$

Gyttja from isolation niveau in basin of raised bog Pärkönsuo $\left(60^{\circ}\right.$ $51^{\prime} \mathrm{N}, 21^{\circ} 40^{\prime} \mathrm{E}$ ), surface alt $12.5 \mathrm{~m}$, depth from 1.9 to $2 \mathrm{~m}$. Coll 1973 with large peat sampler made in USSR and subm 1974 by K Tolonen. Comment: 1st cerealia pollen grains occur in dated horizon, age is ca 400 to $500 \mathrm{yr}$ "too old" when compared with land-uplift chronology based on diatoms (op cit, above).

\section{Su-295. Kozlu, Erbaa}

\section{Turkey}

$4750 \pm 30$

$$
\delta^{13} \mathrm{C}=-23.6 \%
$$

Wood, Kozlu $\left(40^{\circ} 36^{\prime} \mathrm{N}, 36^{\circ} 25^{\prime} \mathrm{E}\right)$, from depth $6 \mathrm{~m}$ in hand-dug pit; coll May 1973 by E P Kuijpers and subm 1973 by J Huhta (Giles \& Kuijpers, 1974).

\section{PALEOZOOLOGIC SAMPLES}

\section{Su-554. Isojoki, SW Finland}

Wood with clear marks of gnawing by beaver (Castor fiber L), from bog Todiston neva $\left(62^{\circ} 02^{\prime} \mathrm{N}, 21^{\circ} 52^{\prime} \mathrm{E}\right)$, surface alt $79 \mathrm{~m}$, depth $1.5 \mathrm{~m}$. Museum sample. Subm 1975 by E Lappalainen (Lappalainen \& Lahti, 1972; 1973).

Su-555. Kirkkonummi, S Finland

$$
2510 \pm 30
$$

Wood, sent to Zool Mus of Helsinki. Sample bears possible gnawing marks by beaver. Subm 1975 by E Lappalainen. 
IV. GEOCHEMICAL SAMPLES

Leaves from birch trees and annual plants from Pitkälampi bog $\left(60^{\circ} 43^{\prime} \mathrm{N}, 24^{\circ} 12^{\prime} \mathrm{E}\right)$, Loppi, S Finland, alt $+110 \mathrm{~m}$. Samples grew on paludifying shore of oligotrophic pond. They were cleaned and washed with distilled water; coll July 1973, Aug 1974, and June 1975 by Aulis Heikkinen (Heikkinen et al, 1974).

\section{Su-296. Loppi, $1-1973$}

Leaves (Carex lasiocarpa), coll July 5, 1973.

\section{Su-297. Loppi, 2-1973}

Leaves (Carex vesicaria), coll July 5, 1973.

\section{Su-298. Loppi, 3-1973}

Leaves (Phragmites communis), coll July 4, 1973.

\section{Su-299. Loppi, 4-1973}

Leaves (Betula odorata), coll July 4, 1973.

\section{Su-438. Loppi, 1-1974}

Leaves (Carex lasiocarpa), coll Aug 10, 1974.

\section{Su-439. Loppi, 2-1974}

Leaves (Carex vesicaria), coll Aug 10, 1974.

\section{Su-440. Loppi, 3-1974}

Leaves (Phragmites communis), coll Aug 10, 1974.

\section{Su-441. Loppi, 4-1974}

Leaves (Betula odorata), coll Aug 10, 1974.

\section{Su-518. Loppi, 1-1975}

Leaves (Carex lasiocarpa), coll July 10, 1975.

\section{Su-519. Loppi, 2-1975}

Leaves (Carex vesicaria), coll July 10, 1975.

$$
\begin{array}{r}
\delta^{14} \mathbf{C}=+\mathbf{4 7 2} \pm \mathbf{9} \% \\
\Delta^{14} \mathbf{C}=+\mathbf{4 6 3} \pm \mathbf{9} \% \\
\delta^{13} C=-21.8 \% \\
\\
\delta^{14} \mathbf{C}=+\mathbf{4 6 4} \pm \mathbf{5} \% \\
\Delta^{14} \mathbf{C}=+\mathbf{4 5 3} \pm \mathbf{5} \% \\
\delta^{13} C=-21.3 \% \\
\\
\delta^{14} \mathbf{C}=+\mathbf{4 7 2} \pm \mathbf{5} \% \\
\Delta^{14} \mathbf{C}=+\mathbf{4 6 0} \pm \mathbf{5} \% \\
\delta^{13} C=-20.9 \%
\end{array}
$$

$$
\begin{array}{r}
\delta^{14} \mathbf{C}=+\mathbf{4 5 5} \pm \mathbf{4} \% \\
\Delta^{14} \mathbf{C}=+\mathbf{4 5 4} \pm \mathbf{4} \% \\
\delta^{13} C=-24.4 \%
\end{array}
$$

$\delta^{14} \mathbf{C}=+417 \pm 5 \%$

$\Delta^{14} \mathbf{C}=+\mathbf{4 1 9} \pm \mathbf{5} \%$ $\delta^{13} C=-27.2 \%$

$$
\begin{array}{r}
\delta^{14} \mathbf{C}=+\mathbf{4 2 3} \pm \mathbf{5} \% \\
\Delta^{14} \mathbf{C}=+\mathbf{4 2 6} \pm \mathbf{5} \% \\
\delta^{13} \mathrm{C}=-28.4 \%
\end{array}
$$

$\delta^{14} \mathrm{C}=+429 \pm 3 \%$

$\Delta^{14} \mathrm{C}=+\mathbf{4 2 9} \pm \mathbf{3} \%$ $\delta^{13} \mathrm{C}=-25.0 \%$

$$
\begin{array}{r}
\delta^{14} \mathbf{C}=+422 \pm 2 \% \\
\Delta^{14} \mathbf{C}=+\mathbf{4 2 6} \pm \mathbf{2} \% \\
\delta^{13} C=-28.8 \%
\end{array}
$$

$\delta^{14} \mathrm{C}=+403 \pm 12 \%$

$\Delta^{14} \mathrm{C}=+404 \pm 12 \%$ $\delta^{1 s} C=-26.7 \%$

$\delta^{14} \mathrm{C}=+422 \pm 10 \%$ $\Delta^{14} \mathrm{C}=+\mathbf{4 2 5} \pm \mathbf{1 0} \%$ $\delta^{13} C=-28.2 \%$ 


\section{Su-520. Loppi, 3-1975}

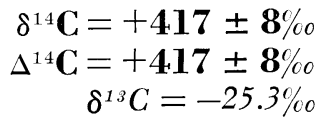

Leaves (Phragmites communis), coll July 10, 1975.

\section{Su-521. Loppi, 4-1975}

$$
\begin{array}{r}
\delta^{14} \mathbf{C}=+\mathbf{3 9 8} \pm \mathbf{5} \% \\
\Delta^{14} \mathbf{C}=+\mathbf{4 0 0} \pm \mathbf{5} \% \circ \\
\delta^{13} C=-27.4 \% \circ
\end{array}
$$

Leaves (Betula odorata), coll July 10, 1975.

\section{REFERENCES}

Giles, D L and Kuijpers, E P, 1974, Stratiform copper deposit, Northern Anatolia, Turkey: evidence for Early Bronze I (2800 BC) mining activity: Science, v 186, p 823-825.

Heikkinen, Aulis, 1971, Geological Survey of Finland radiocarbon measurements V: Radiocarbon, v 13, p 432-441.

- 1975, Radiohiilimenetelmän käytöstä moreenin sisältämän vanhan hiiliaineksen ajoituksessa (Radiocarbon dating of old charcoal contained in till): Geologi, v 27, p 51-53.

Heikkinen Aulis, Koivisto, Anna-Kristiina, and Äikää, Osmo, 1974, Geological Survey of Finland radiocarbon measurements VI: Radiocarbon, v 16, p 252-268.

Hirvas, Heikki, Kujansuu, Raimo, and Tynni, R, 1976, Till stratigraphy in Nortern Finland: Project 73/1/24 "Quaternary Glaciations in the Northern Hemisphere", rept no. 3, p 256-273.

Lahermo, Pertti, Valovirta, Veikko, and Särkioja, Aarno, 1977, The geobotanical development of spring-fed mires in Finnish Lapland: Geol Survey Finland, Bull 287, in

press.
Lappalainen, Eino and Lahti, Seppo, 1972, Postglacial evidence of the occurrence of the beaver (Castor fiber $L$ ) in eastern Finland: Annals Zool Fennici 9, p 139-140. 1973, Majavan jääkauden jälkeisestä historiasta Suomessa (Subfossil remnants as an evidence of the occurrence of the beaver [Castor fiber $L$ ] in Finland): Geologi, v 25, p 34-35.

Kotilainen, Mauno J, 1953, Hiekkakerrosten alle peittynyt muinaissuo Kuopion yläkaupungilla: Geologi, v 5, p 59-61.

Kukkonen, Esa and Tynni, Risto, 1970, Die Entwicklung des Sees Pyhäjärvi in Südfinnland im Lichte von Sedimentund Diatomeenuntersuchungen: Acta Bot Fennica 90, p $1-30$.

Kujansuu, Raimo, 1975, Marrasjärven interstadiaalinen harju Keski-Lapissa (Interstadial esker at Marrasiärvi, Finnish Lapland): Geologi, v 27, p 45-50.

Niemelä, Jouko and Tynni, Risto, 1975, Alustava tiedonanto Etelä-Pohjanmaan moreeninalaisten harjujen iästa (A preliminary report on the age of the submorainic eskers in Southern Ostrobothnia): Geologi, v 27, no. 9-10, p 15-16.

Rainio, Heikki and Lahermo, Pertti, 1976, Observations on dark grey basal till in Finland: Geol Soc Finland Bull, no. 48, p 137-152.

Tanskanen, Heikki, 1975, Tiedoksianto moreeninalaisesta turvekcrrostumasta KeskiLapissa (Notice on a peat-layer underlying till in Middle-Lappland, Finland): Geologi, v 27, p 54-55.

Tolonen, Kimmo, Siiriäinen, Ari, and Thompson, Roy, 1975a, Espoon Lojärven pohjakerrostumien tuulkinta ja paleomagneettinen ajoitus (Interpretation and paleomagnetic dating of sediments in Lake Lojärvi, S Finland): Geologi, v 27, p $97-102$.

P 1975 b, Prehistoric field erosion sediment in Lake Lojärvi, S Finland and its palcomagnetic dating: Annals Bot Fennici 12, p 161-164.

Tolonen, K and Ruuhijärvi, R, 1976, Standard pollen diagrams from the Salpausselkä regions of Southern Finland: Annals Bot Fennici 13, p 155-196.

Tolonen, K Siiriäinen, A, and Hirviluoto, A-L, 1977, On the Iron age agriculture in SW Finland: Finskt Mus, 1975 (in press). 